\title{
Développement de nouvelles applications pour l'utilisation de protéases en œnologie
}

\section{Development of new applications for the use of proteases in the enological context}

\author{
J. Ducruet ${ }^{1,2}$, R. Cahuzac ${ }^{2}$, A.-C. Silvestri ${ }^{2}$, J. Roeslé2 , B. Bach ${ }^{2}$, M. Cléroux ${ }^{2}$, C. Koestel ${ }^{3}$, F. Laurenzini ${ }^{3}$, et J. Roesti ${ }^{3}$ \\ ${ }^{1}$ Inter-Rhône, 2260 route du Grès, 84100 Orange, France \\ ${ }^{2}$ Changins, Haute école de viticulture et d'œnologie, Route de Duillier 50, 1260 Nyon, Suisse \\ ${ }^{3}$ Agroscope, Route de Duillier 50, 1260 Nyon, Suisse
}

\begin{abstract}
Résumé. Depuis les années 70, les préparations enzymatiques sont des auxiliaires technologiques d'origine biologique de plus en plus appréciées pour l'aide qu'elles apportent dans les différentes étapes de la vinification (Débourbage, pressurage, macération, filtration et stabilisation microbiologique). Les activités utilisables en œnologie sont décrites par le règlement européen 1493/1999 et les résolutions œnologiques 11-18/2004 de l'OIV. A ce jour, les protéases ne font pas partie des activités enzymatiques autorisées car jugées inefficaces dans le milieu hostile qu'est le vin. Plus récemment, il a été démontré que l'utilisation d'une aspergillopepsines couplée de la flash pasteurisation permettait l'hydrolyse complète des protéines thermoinstables des vins blancs à l'échelle pilote et industrielle.

L'objectif de ce travail est d'explorer de nouvelles applications œnologiques à l'utilisation de protéases. Pour cela, 4 protéases industrielles ont été testées sur l'amélioration de la filtrabilité des vins et l'élevage sur lies d'un vin rouge et un vin blanc. Ce travail a permis de mettre en évidence que l'utilisation de protéase pouvait améliorer sensiblement la filtrabilité des vins. L'amélioration observée est plus importante avec un test de filtrabilité (Vmax sur membrane $0.45 \mu \mathrm{m}$ ) qu'en filtration tangentielle (membrane céramique $0.2 \mu \mathrm{m}$ ). Enfin, en l'absence de flash pasteurisation les protéases ne semblent pas avoir d'effet sur la filtrabilité ou l'élevage sur lies des vins.
\end{abstract}

Mots clés : Enzyme, Protéase, filtrabilité, filtration tangentielle, vin.

\begin{abstract}
Since the 1970s, enzymatic preparations have been used as technological aids of biological origin. They are increasingly appreciated for the help they provide in the different stages of winemaking (settling, pressing, skin contact, filtration and microbiological stabilization). The enzymatic activities that can be used in oenology are described by the European regulation 1493/1999 and oenological resolutions 11-18/2004 of the OIV. To date, proteases are not part of the enzymatic activities authorized because they are considered inefficient in the hostile environment that is wine. More recently, it has been shown that the use of flash pasteurization coupled with aspergillopepsins allows the complete hydrolysis of the thermo-unstable proteins of white wines at pilot and industrial scale.

The goal of this work is to explore new oenological applications for the use of proteases. For this, 4 industrial proteases were tested on the improvement of the filterability and the aging on the lees of a red wine and a white wine. This work highlight that the use of protease could significantly improve the filterability of the wines. The improvement observed is greater with a filterability test (Vmax on membrane $0.45 \mu \mathrm{m})$ than with cross flow filtration (ceramic membrane $0.2 \mu \mathrm{m}$ ). Finally, in the absence of flash pasteurization proteases do not seem to have any effect on the filterability or aging on lees of wines.
\end{abstract}

Key words: Enzyme, Protease, filtrability, Crossflow filtration, wine.

\section{Introduction}

Les préparations enzymatiques sont apparues sur le marché œnologique dans les années 70. Elles sont de plus en plus appréciées pour l'aide qu'elles apportent dans les différentes étapes de la vinification. Elles constituent, avec les levures sélectionnées et les bactéries, des outils biotechnologiques de l'œnologie moderne. Elles ne sont pas considérées comme des additifs mais comme des auxiliaires technologiques. Les enzymes sont de puissants catalyseurs biologiques très spécifiques. Elles vont accélérer une réaction précise sur un substrat bien déterminé. Ainsi, elles rendent possible la diminution du temps ou de l'intensité des traitements mécaniques utilisés lors de la macération, du pressurage, de la clarification ou de la filtration [1]. 
Les préparations enzymatiques utilisées en vinification sont obtenues par fermentation de cultures de souches sélectionnées de l'espèce Aspergillus $S p$ pour les pectinases et les glucosidases et de l'espèce Trichoderma harzianum pour les glucanases. Le lysozyme est quant à lui extrait directement du blanc d'œuf.

Les enzymes sélectionnées pour l'œnologie sont des mélanges de différentes activités enzymatiques. Elles sont classées suivant leurs activités principales mais aussi en fonction de certains critères de purification. Cinq grandes familles de préparation sont actuellement utilisées en œnologie : les enzymes de macération, de clarification, de filtration ou d'élevage, de révélation des arômes terpéniques et antibactériennes.

Les activités utilisables en œnologie sont décrites par le règlement européen 1493/1999 et les résolutions œnologiques 11-18/2004 de l'OIV. A ce jour, les protéases ne font pas partie des activités enzymatiques autorisées. Or, les protéases acides trouvent leur application dans diverses industries, y compris la médecine, les industries pharmaceutiques, du cuir, des aliments et des boissons $[2,3]$. Au sein de l'industrie des boissons, ces enzymes ont particulièrement attiré l'attention pour leur potentiel à dégrader les protéines formant un trouble. Elles sont aussi largement utilisées pour la farine destinée à la boulangerie et à la fabrication de fromage [2].

Aussi séduisante qu'elle puisse être, l'idée d'utiliser des protéases en œnologie est toutefois confrontée aux conditions physico-chimiques du jus de raisin et du vin, hostiles pour ces enzymes. Il existe plusieurs facteurs et composés du vin qui vont inhiber les activités enzymatiques. Un groupe de chercheurs a étudié l'effet des inhibiteurs de l'activité des protéases [4]. L'activité de l'enzyme contre un tripeptide synthétique a été évaluée dans un milieu acide ( $\mathrm{pH} 3.2$ ) en présence de 0.12 et $18 \%$ (v/v) d'éthanol. L'éthanol a seulement un effet inhibiteur sur l'activité enzymatique à une concentration de $18 \%$ $(\mathrm{p} / \mathrm{v})$. De plus, l'activité a été également évaluée dans un vin ( $\mathrm{pH} 3.2)$ avec de l'éthanol à $12 \%(\mathrm{v} / \mathrm{v})$ et avec addition de $\mathrm{SO}_{2}$ libre, de tanins de pellicule et de tanins de pépins. Le $\mathrm{SO}_{2}$ libre a un effet inhibiteur significatif sur l'activité enzymatique à la plus faible concentration testée $\left(10 \mathrm{mg} / \mathrm{L} \mathrm{SO}_{2}\right.$ libre) et les deux sources de tanins exercent des effets négatifs similaires sur l'activité de l'enzyme. Les polyphénols sont, dans le vin rouge en quantité (2 à $5 \mathrm{~g} / \mathrm{L}) 100$ fois supérieure aux quantités supposées de protéines (20 à $100 \mathrm{mg} / \mathrm{L}$ ) [5]. De nombreux travaux ont montré que les tanins s'associent avec les protéines par des liaisons hydrogènes ainsi que des interactions hydrophobes. D'autres études ont révélé qu'il y a des facteurs liés à la matrice du vin comme par exemple, le $\mathrm{pH}$, la concentration en éthanol, l'acidité [6] et les polysaccharides [7].

Malgré la présence de $\mathrm{SO}_{2}$, d'éthanol, un $\mathrm{pH}$ acide et des tanins il est possible d'obtenir des activités enzymatiques même dans un vin rouge. Depuis les années 1990, des études ont montré que l'utilisation des protéases dans le vin est un moyen efficace de réduire la formation de voile protéique sans nuire à la qualité du vin [8,9]. Cependant l'enzymage doit être associé à un traitement thermique afin de dénaturer les protéines thermo-instables avant leur dégradation. Cela est en accord avec l'étude de Conterno [10], qui affirme que, sans dénaturation préalable, les protéines formant un trouble sont résistantes à la protéolyse. En 2003, Pocock et al. [9] ont démontré que la combinaison $\mathrm{du}$ traitement thermique et des enzymes protéolytiques réduisait l'utilisation de la bentonite de 50 à $70 \%$ sans affecter le profil sensoriel du vin final. Basées sur le succès prometteur des protéases pour éliminer le trouble, les recherches sur les protéases aspartiques pour des applications en œnologie se sont intensifiées et étendues. Benucci et al. [4] ont étudié l'activité de la bromélaïne libre (à partir d'ananas) dans des vins blancs. Afin d'évaluer l'activité, un tripeptide chromogène synthétique a été ajouté au vin. Les échantillons ont été par la suite incubés à $20^{\circ} \mathrm{C}$ pendant $24 \mathrm{~h}$ et le trouble potentiel a été déterminé par test thermique. Globalement, il en ressort qu'il faut une quantité très importante $(10 \mathrm{~g} / \mathrm{L})$ de bromélaïne pour réduire de $70 \%$ le potentiel trouble du vin. L'exposition à la chaleur dénature les protéines formant un trouble permettant l'accès aux enzymes protéolytiques afin d'hydrolyser les protéines en peptides plus petits. Malgré ces résultats encourageants, il a été conclu qu'une approche plus efficace des protéases était nécessaire. Au cours du premier stade de la casse, des températures élevées induisent le dépliement des protéines [11]. Comme une longue exposition à la chaleur peut nuire à la qualité du vin, la flash-pasteurisation couplée à l'utilisation de protéases aspartiques d'origine fongiques (aspergillopepsines) a été identifiée comme la solution la mieux adaptée. En effet, l'addition seule d'apergillopepsine entraîne une réduction de $20 \%$ des protéines du vin, mais couplée à une flash-pasteurisation ( $75^{\circ} \mathrm{C}$ pendant $1 \mathrm{~min}$ ), elle peut dégrader complètement les protéines des vins de Chardonnay et de Sauvignon Blanc [12]. Le traitement combiné de l'addition de protéase et de Flash Pasteurisation s'est même avéré efficace à l'échelle industrielle [13], et les mêmes aspergillopepsines ont été approuvées pour la vinification en Australie et en

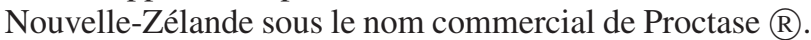
L'objectif de la Flash Pasteurisation est double. Elle permet à la fois d'augmenter l'activité protéasique mais également de dénaturer les protéines thermo-instables afin de les rendre plus accessibles à l'hydrolyse enzymatique.

L'objectif de ce travail est d'explorer de nouvelles applications œnologiques à l'utilisation de protéases. Pour cela 4 protéases industrielles ont été testées sur l'amélioration de la filtrabilité des vins et l'élevage sur lies d'un vin rouge et un vin blanc.

\section{Matériel et méthode}

\subsection{Incidence des enzymes et de la flash pasteurisation sur la filtrabilité des vins}

Des enzymes commerciales sont testées: E1 (poudre), E2 (liquide), E3 (poudre), E4 (poudre). Cet essai est réalisé sur un vin blanc (Chasselas, Romand, millésime 2017, Schenk SA Rolle, code B) et un vin rouge (Gamay, Romand, millésime 2017, Schenk SA Rolle, code R).

Un volume de $2500 \mathrm{~L}$ de vin est réparti de manière homogène dans dix cuves inox de $250 \mathrm{~L}$ à chapeau flottant. Chacune des quatre enzymes; E1 à E4 est ajoutée à $0.1 \mathrm{~g} / \mathrm{L}$ ou $0.1 \mathrm{~mL} / \mathrm{L}$ dans deux cuves et homogénéisées. Les deux dernières cuves serviront de témoin (code E0). La première moitié des cuves est flash-pasteurisée $\left(70^{\circ} \mathrm{C}\right.$, 1 min, code FB), l'autre non (code B). Les rouges sont 
Tableau 1. Composition des vins des essais.

\begin{tabular}{|l|l|l|}
\hline Analyse WineScan & Chasselas 2017 & Gamay 2017 \\
\hline Ethanol ( \% Vol.) & 12.27 & 13.03 \\
\hline $\mathrm{pH}$ & 3.6 & 3.6 \\
\hline Acidité totale $(\mathrm{T} . \mathrm{g} / \mathrm{l})$ & 3.87 & 5.27 \\
\hline Acide acétique $(\mathrm{g} / \mathrm{l})$ & 0.58 & 0.72 \\
\hline Acide tartrique $(\mathrm{g} / \mathrm{l})$ & 1.77 & 2.29 \\
\hline Acide malique $(\mathrm{g} / \mathrm{l})$ & $<0.3$ & $<0.3$ \\
\hline Acide lactique $(\mathrm{g} / \mathrm{l})$ & 1.73 & 2 \\
\hline Glucose-Fructose $(\mathrm{g} / \mathrm{L})$ & 1.66 & $<1$ \\
\hline Glycérol $(\mathrm{g} / \mathrm{l})$ & 5.9 & 9.16 \\
\hline Extrait sec $(\mathrm{g} / \mathrm{l})$ & 18.54 & 28.26 \\
\hline
\end{tabular}

pasteurisés $\left(60^{\circ} \mathrm{C}, 1 \mathrm{~min}\right.$, code $\mathrm{FR}$ ) ou non (code R). Une variante enzymée après flash-pasteurisation est également réalisée sur les rouges (code FRE2P). Les cuves sont placées à $16^{\circ} \mathrm{C}$. L'activité protéasique, la turbidité et le Vmax sont suivis durant 4 semaines. A la fin de l'essai, les vins sont analysés (stabilité protéique, WineScan, électrophorèse SDS-PAGE, analyses sensorielles). Des essais de filtrabilité sur prototype de filtre tangentiel sont également réalisés.

Pour la flash pasteurisation et afin de ne pas dénaturer les qualités organoleptiques du vin, il est important d'avoir un profil thermique précis. Le montage est constitué d'un premier échangeur branché sur une source d'eau chaude tournant en boucle sur l'échangeur thermique $\left(80^{\circ} \mathrm{C}\right.$ pour les vins blancs et $70^{\circ} \mathrm{C}$ pour les rouges) et dont la température est maintenue par deux générateurs de vapeur. Le vin à pasteuriser est chauffé en 20 secondes en passant par ce premier échangeur $\left(70^{\circ} \mathrm{C}\right.$ pour les vins blancs et de $60^{\circ} \mathrm{C}$ pour les vins rouges). La zone d'arrêt et constitué par une conduite de volume connu et isolée. Le vin est maintenu à cette température durant 1 minute. Le refroidissement se fait, également en 20 secondes, par l'intermédiaire d'un second échangeur thermique branché sur le réseau d'eau froide. Le contrôle des températures de chauffage et de refroidissement se fait toutes les 5 minutes à l'aide d'un thermomètre infrarouge, afin de s'assurer que la température souhaitée est maintenue. La flash pasteurisation des vins se fait en continue avec un passage d'eau clair entre chaque varientes.

\subsection{Incidence des enzymes sur l'élevage sur lies}

Un mout de Chasselas est réparti en cinq cuves de $200 \mathrm{~L}$ et $3 \%$ de lies de chasselas sont ajoutés. Dans chaque cuves une enzyme E1 à E4 est ajoutée dans les cuves 1 à 4 . Une cuve témoin sans enzyme (E0) est réalisée. Les cuves sont identifiées LBE0, LBE1, LBE2, LBE3, LBE4. Le même protocole est réalisé avec du vin et des lies de Gamay. Les cuves sont identifiées LRE0, LRE1, LRE2, LRE3, LRE4. Les lies sont bâtonnées deux fois par semaines pendant 5 semaines et l'activité protéasique, la turbidité et le $V_{\max }$ de toutes les cuves sont suivies toutes les semaines. Les vins sont ensuite filtrés sur un filtre tangentiel.

\subsection{Analyse de l'activité protéasique résiduelle des vins}

La méthode de dosage de l'activité protéase résiduelle dans les vins utilise une caséine conjuguée à un colorant azoïque. L'hydrolyse de cette azocaséine libère le colorant azoïque dans le milieu où il est détecté par absorbance à 440 nm [14].

Pour cela, préparer une solution à $0.5 \%$ d'azocaséine dissout dans le tampon McIlevaine. Préparer la solution A contenant $900 \mu \mathrm{L}$ d'azocaséine à $0.5 \%$ auxquels on ajoute $100 \mu \mathrm{L}$ du vin dont on souhaitait mesurer l'activité protéasique. Vortexer. Prélever $300 \mu \mathrm{L}$ de cette solution A au temps T0 dans lesquels on ajoute $300 \mu \mathrm{L}$ de TCA. Placer le reste de la solution $\mathrm{A}$ à $40^{\circ} \mathrm{C}$ pendant 60 minutes. Pour les temps T0 et T60 vortexer et centrifuger pendant 10 minutes à $13000 \mathrm{~g}$. Mesurer l'absorbance des échantillons à $450 \mathrm{~nm}$ sur lecteur microplaque.

\subsection{Calcul de $V_{\max }$}

La filtrabilité est évaluée par mesure du $\mathrm{V}_{\max }$ ou volume de filtration maximum au colmatage. Le volume de vin écoulé à travers une membrane possédant des pores de $0.45 \mu \mathrm{m}$ de diamètre est mesuré après 2 et 5 minutes sous une pression de 1 bar. Le $\mathrm{V}_{\max }(\mathrm{ml})$ est calculé selon la formule suivante :

$$
V_{\max }=\frac{(5-2)}{\frac{5}{V 5}-\frac{2}{V 2}}=\frac{3 x(V 5 x V 2)}{5 V 2-2 V 5}
$$

$\mathrm{V} 2=$ volume de vin filtré à $2 \mathrm{~min}(\mathrm{ml})$

$\mathrm{V} 5=$ volume de vin filtré à $5 \mathrm{~min}(\mathrm{ml})$.

\subsection{Mesure de la turbidité}

L'unité de turbidité utilisée est le: NTU-Nephelometric Turbidity Unit (unité de turbidité néphélométrique) qui correspond à la mesure de la lumière diffusée par une suspension étalon de silice dans un nephelometre $(\mathrm{ACH}$ 2100Q).

\subsection{Utilisation d'un prototype de filtre tangentiel}

La filtration tangentielle est réalisée sur une membrane en céramique de $0.2 \mu \mathrm{m}$ de diamètre de pores et $75.4 \mathrm{~cm}^{2}$ de surface filtrante (Inside Céram ${ }^{\mathrm{TM}}$, Tami Industries, France). Le prototype de paillasse utilisé (TAMI Industries, France) et thermorégulé et a une capacité de $10 \mathrm{~L}$. La pression transmembranaire de filtration et réglée à 1.35 bar $(\mathrm{PTM}=($ Pentrée + Psortie $) /$ 2 - contre pression).

\subsection{Analyse des vins au WineScan}

Le WineScan FOSS est un analyseur multi-paramètres de la composition chimique du vin. Les mesures sont réalisées à l'aide de la spectroscopie infrarouge par transformée de Fourier (IRTF) permettant d'analyser un échantillon liquide. Il nous permettra d'analyser des paramètres tels que le $\mathrm{pH}$ [Ethanol \%], [Acide Malique], [Acide Tartrique], [Acidité volatile], [Acidité Totale], [Glucose + Fructose].

\subsection{Détermination des caractéristiques chromatiques des vins}

Ces mesures sont réalisées sur un spectrophotomètre CM-5 KONICA MINOLTA. L'échelle de mesure est appelée 
« échelle CIE 1976 - L*, a*, b* » ou plus souvent abrégée « échelle CIELAB ». Ce système de couleur ou espace CIELab se fonde sur une représentation cartésienne séquentielle ou continue sur 3 axes orthogonaux $L^{*}, a^{*}$ et $b^{*}$. La coordonnée $L^{*}$ désigne la clarté $\left(L^{*}=0\right.$ noir et $\mathrm{L}^{*}=100$ incolore), $\mathrm{a}^{*}$ désigne la composante de couleur rouge/vert $\left(\mathrm{a}^{*}>0\right.$ rouge, $\mathrm{a}^{*} 0$ jaune, $\mathrm{b}^{*}<0$ bleu).

Différence colorimétrique globale entre deux vins :

$$
\Delta \mathrm{E}^{*}=\sqrt{\left(\Delta \mathrm{L}^{*}\right)^{2}+\left(\Delta \mathrm{a}^{*}\right)^{2}\left(\Delta \mathrm{b}^{*}\right)^{2}}
$$

Différence de ton entre deux vins :

$$
\Delta \mathrm{H}^{*}=\sqrt{\left(\Delta \mathrm{E}^{*}\right)^{2}-\left(\Delta \mathrm{L}^{*}\right)^{2}\left(\Delta \mathrm{C}^{*}\right)^{2}} .
$$

\subsection{Analyse des protéines du vin par gel d'électrophorèse (SDS-PAGE)}

Les protéines du vin blanc sont précipitées à $-20^{\circ} \mathrm{C}$ dans de l'éthanol $(1 / 10, v / v)$. Celles du vin rouge sont obtenues par émulsion méthanol/chloroforme et analysées sur gel d'électrophorèse SDS-PAGE [15].

\subsection{Analyses sensorielles}

Les analyses sensorielles sont réalisées en verre INAO dans une salle de dégustation respectant la norme ISO 8589 par le panel expert de l'école de Changins (Suisse).

Des analyses de napping et des tests triangulaires sont réalisés et interpété par le logiciel Fizz (Biosystemes, Dijon, France).

Napping : Les juges qui doivent positionner les vins présentés sur une «nappe ». Plus les vins se ressemblent, plus ils sont positionnés proches sur la nappe et plus ils sont différents, plus ils sont éloignés. Chaque juge doit réaliser son propre positionnement en 2 dimensions en fonction des caractéristiques sensorielles qui lui semblent importantes pour différencier les vins. Le dégustateur peut annoter quelques mots caractérisant les différents groupes de vins. On peut trier ce vocabulaire afin de limiter les descripteurs.

Test triangulaire : Ce test permettra de voir s'il existe des différences perceptives entre deux vins. Pour cela 3 échantillons sont présentés à un sujet, deux sont identiques et le sujet doit déterminer quel échantillon est différent. Pour mettre en place ce test, l'ordre de présentation des échantillons est équilibré et un codage à 3 chiffres est utilisé.

\section{Résultats et discussion}

\subsection{Incidence des enzymes sur l'élevage sur lies}

En l'absence de traitement thermique il n'a pas été possible d'observer une influence des enzymes sur les principales caractéristiques des vins élevés sur lies (Résultats non présentés).

\subsection{Incidence des enzymes et de la flash pasteurisation sur la filtrabilité des vins}

\subsubsection{Turbidité}

La Fig. 1 présente la turbidité en NTU des vins quatre semaines après l'enzymage.
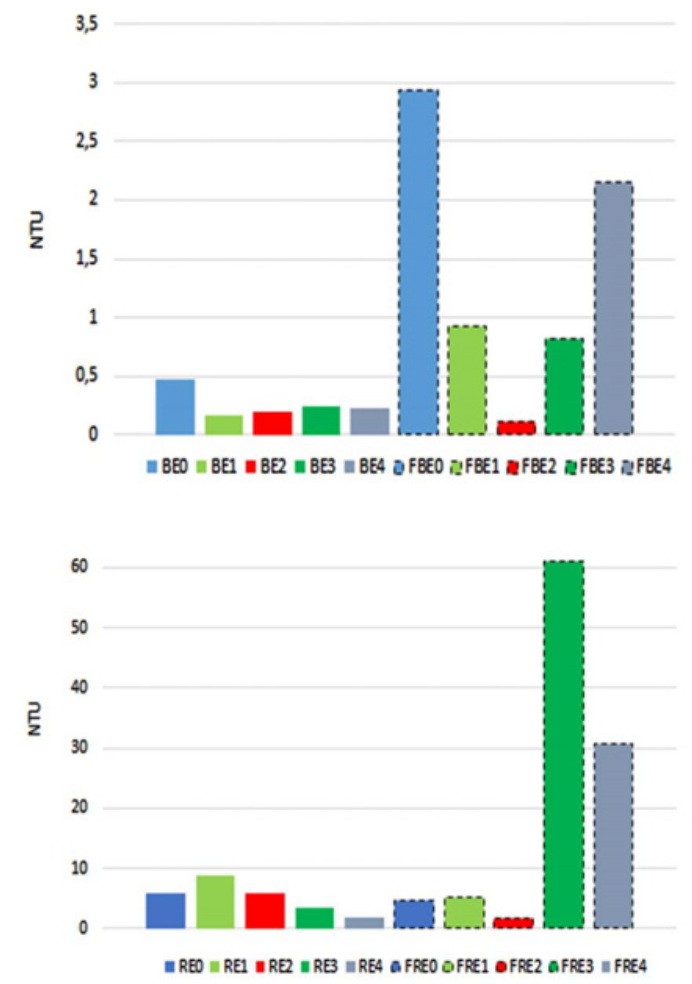

Figure 1. Incidence des enzymes et de la flash pasteurisation sur la filtration tangentielle des vins blancs (B : Chasselas 2017 ; F : Flash Pasteurisation; E1 à E4 : enzymes 1 à 4, E0 sans enzyme).

Pour le Chasselas (Fig. 1), la flash pasteurisation a augmenté la turbidité des vins. La température a induit une dénaturation des protéines qui forment un trouble lors du refroidissement [11]. Les 4 enzymes ont permis de limiter partiellement ce trouble. Les échantillons flash pasteurisé avec l'enzyme E2 (FBE2 et FE2) permettent d'obtenir les turbidités les plus basses de toutes les variantes et quel que soit la couleur du vin. La combinaison enzyme E2 et flash pasteurisation permet donc une clarification plus rapide des vins.

\subsection{2. $V_{\max }$}

La filtrabilité des vins est mesurée après 4 semaines d'élevage. Plus l'indice sera élevé, meilleure sera la filtrabilité sur membrane $0.45 \mu \mathrm{m}$. Hormis avec l'enzyme E2, la Flash Pasteurisation a tendance à faire baisser le $\mathrm{V}_{\max }$. La dénaturation thermique des protéines rend la filtration plus difficile et augmente le colmatage de la membrane. Par contre, l'indice $\mathrm{V}_{\max }$ augmente d'un facteur 4 entre le témoin rouge non flash pasteurisé et le vin flash pasteurisé $(\mathrm{RE} 0<\mathrm{FRE} 2)$. Cette augmentation est également visible sur les $\mathrm{V}_{\max }$ des vins blancs mais celleci est plus modérée et passe de 149 pour BE0 à 233 pour FBE2.

Si l'on considère les vins sans flash pasteurisation, l'indice $\mathrm{V}_{\max }$ est deux fois plus élevé pour le vin RE4. D'autres activités possiblement présentent dans cette préparation (pectinases et $\beta$-glucanases) peuvent expliquer ce phénomène. Enfin, l'ajout de l'enzyme avant la Flash Pasteurisation permet de meilleures performances qu' après flash pasteurisation (FRE2 > FRE2P). Cela est en accord avec l'étude de Conterno [10], qui affirme que, sans dénaturation préalable, les protéines formant un trouble 

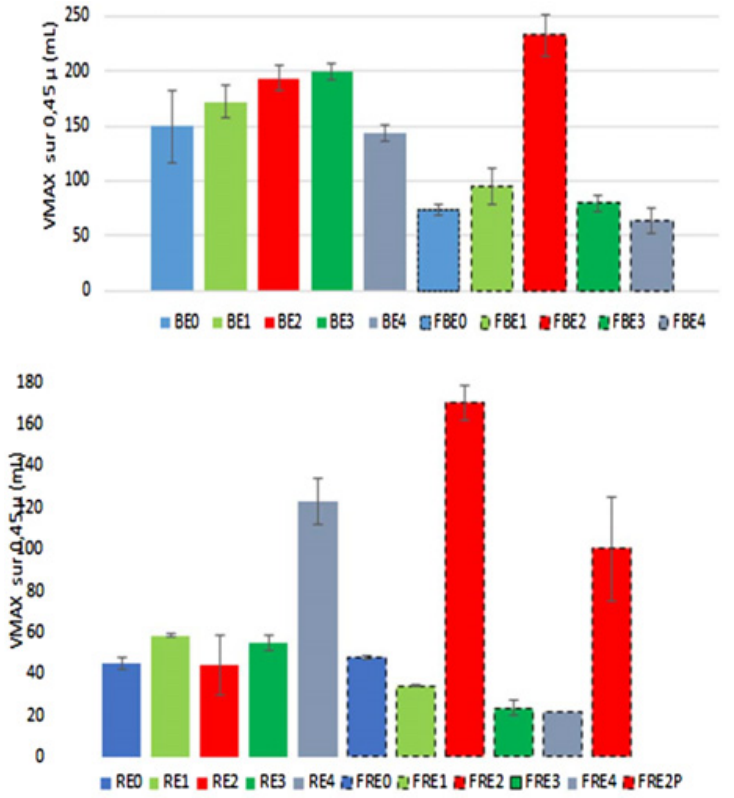

Figure 2. Incidence des enzymes et de la flash pasteurisation sur la filtration tangentielle des vins rouges (R : Gamay 2017; F : Flash Pasteurisation; E1 à E4 : enzymes 1 à 4, E0 sans enzyme).

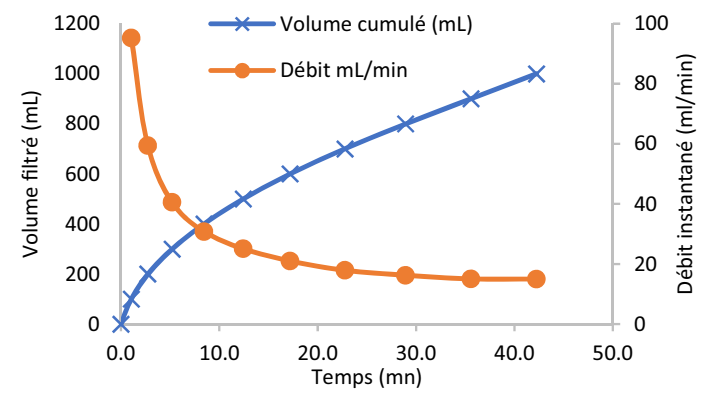

Figure 3. Evolution des volumes filtrés et des débits instantanés lors de la filtration tangentielle du vin blanc témoin BE0.

sont résistantes à la protéolyse et les résultats de Marangon [12] qui indiquent que l'ajout des protéases avant la flash pasteurisation donne de meilleures performances qu'après.

Le fait que les $\mathrm{V}_{\text {max }}$ de RE2 $<$ FRE2P $<$ FRE2 nous indique 2 effets combinés de la température. Elle dénature les protéines du vin, les rendant plus accessibles à l'hydrolyse et améliore la performance intrinsèque de l'enzyme E2.

\subsubsection{Incidence sur la filtration tangentielle}

La Fig. 3 montre l'évolution des volumes filtrés et des débits de filtration dans le temps. Sur ce vin, colmatage de la membrane céramique intervient au bout d'une dizaine de minutes soit entre 0 et $500 \mathrm{ml}$ de vin filtré.

Les vins des essais sont comparés entre eux en fonction du débit moyen de filtration en $\mathrm{mL} / \mathrm{min}$ de 0 à $500 \mathrm{~mL}$ et de 500 à $1000 \mathrm{~mL}$ pour les vins blancs (Fig. 4). Les vins rouges étant beaucoup plus colmatant seul les débits moyens de 0 à $500 \mathrm{ml}$ ont pu être mesuré (Fig. 5).

Sur vin blanc (Fig. 4) et en l'absence de Flash pasteurisation, les enzymes E1 et E2 n'affecte pas la filtrabilité. Dans ces mêmes conditions les enzymes E3 et E4 réduisent la filtrabilité.

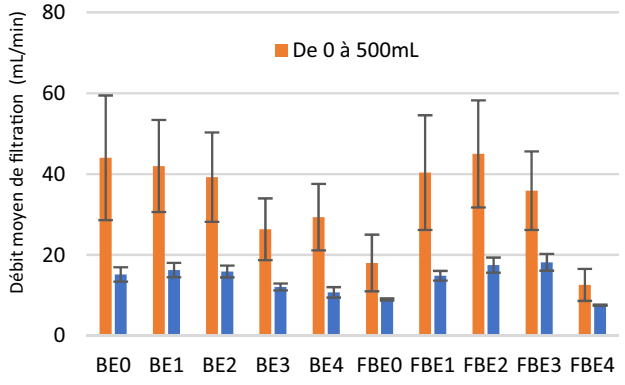

Figure 4. Incidence des enzymes et de la flash pasteurisation sur la filtration tangentielle des vins blancs (B : Chasselas 2017; F : Flash Pasteurisation; E1 à E4 : enzymes 1 à 4, E0 sans enzyme).

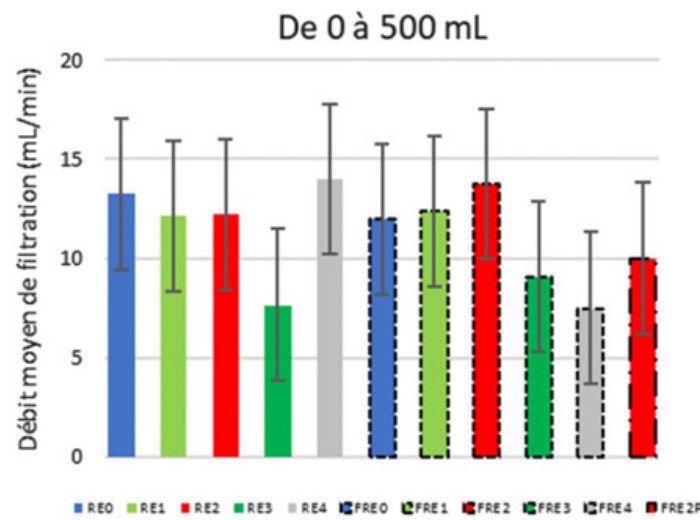

Figure 5. Incidence des enzymes et de la flash pasteurisation sur la filtration tangentielle des vins rouges (R : Gamay 2017; F : Flash Pasteurisation; E1 à E4 : enzymes 1 à 4, E0 sans enzyme).

Toujours sur le vin blanc et en l'absence d'enzyme (Fig. 4), le débit moyen de filtration est significativement réduit par la Flash Pasteurisation (BE0 $\gg$ FBE0). Cette tendance confirme les résultats obtenus lors du test de filtrabilité $\mathrm{V}_{\max }$. La dénaturation des protéines lors de la flash pasteurisation entraine une augmentation du colmatage. Cependant, l'utilisation des enzymes E1, E2 et E3 permettent de compenser cette baisse. Les meilleures performances semblent être observées sur la variante FBE2 sans toutefois que cela soit significatif par rapport au témoin (Fig. 4 : BE0 $\leq$ FBE2).

Cette amélioration est nettement moins visible que sur le test de filtrabilité $\mathrm{V}_{\max }$ sur ce prototype de filtre tangentiel. FBE3 se comporte également très différemment entre ces deux modes de filtration. Ceci peut s'expliquer par les principes de filtration différents entre ces deux méthodes (filtration frontale sur $0.45 \mu \mathrm{m}$ et tangentielle sur $0.2 \mu \mathrm{m})$.

Pour les vins rouges (Fig. 5), l'impact des enzymes couplées à la Flash Pasteurisation est modéré sur le débit moyen de filtration. L'enzyme E3 semble réduire les débits de filtration avec ou sans Flash Pasteurisation.

\subsubsection{Activité protéasique résiduelle}

La Fig. 6 montre l'activité protéasique résiduelle dans vins blancs de l'essai avant et après filtration tangentielle. Seule les enzymes E2 et E3 possèdent une activité protéasique mesurable dans les vins. La filtration tangentielle induit une diminution généralisée de l'activité protéasique résiduelle. D'autre part, la flash pasteurisation réduit considérablement cette activité résiduelle. Finalement, en 

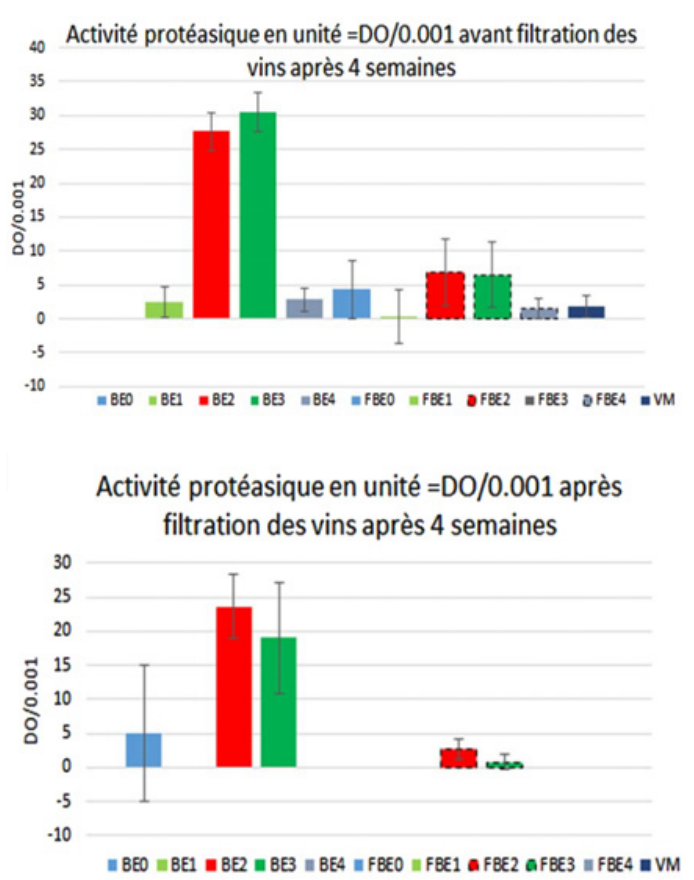

Figure 6. Activités protéases résiduelles des vins blancs (Chasselas, 2017) avant (en haut) et après filtration (en bas), après 4 semaines de contact.

couplant la Flash-Pasteurisation et la filtration tangentielle, la méthode par le dosage de l'azocaséine ne nous permet pas de mesurer une activité protéasique résiduelle significative dans les vins. Il semble donc que la flash pasteurisation soit capable d'améliorer l'efficacité de l'activité protéasique tout en dénaturant les protéases. Il est possible que la cinétique de dénaturation des protéases soit lente et que la dénaturation intervienne après la flash pasteurisation et l'hydrolyse des protéines du vin.

Pour les vins rouges (Fig. 7) la aussi seule les enzymes E2 et E3 possède une activité protéasique mesurable dans le vin. La aussi la flash pasteurisation réduit l'activité protéasique. Par contre si l'enzyme E2 est ajouté après flash pasteurisation sa dénaturation est bien moindre. Finalement la filtration tangentielle réduit l'activité protéasique de tous les vins qu'ils aient été traités en flash pasteurisation ou non. L'hypothèse est que les protéases forment un complexe avec les tanins du vin plus facilement retenu par le filtre tangentiel.

La combinaisons flash pasteurisation et filtration tangentielle donne des activités protéasiques résiduelles inferieures au témoin sans enzyme donc pouvant être considérées comme nulles.

\subsubsection{Caractéristique chromatique des vins}

La coordonnée $\mathrm{L}^{*}$ désigne la clarté $\left(\mathrm{L}^{*}=0\right.$ noir et $\mathrm{L}^{*}=$ 100 incolore), $\mathrm{a}^{*}$ et $\mathrm{b}^{*}$ sont deux gammes de couleurs allant respectivement du vert au rouge et du bleu au jaune avec des valeurs allant de -120 à +120 .

La comparaison en blanc et en rouge entre les vins témoin sans enzyme et les vins pasteurisés et enzymés avec E2 permet de voir qu'il n'y aucun impact sur les caractéristiques chromatiques des vins testés.

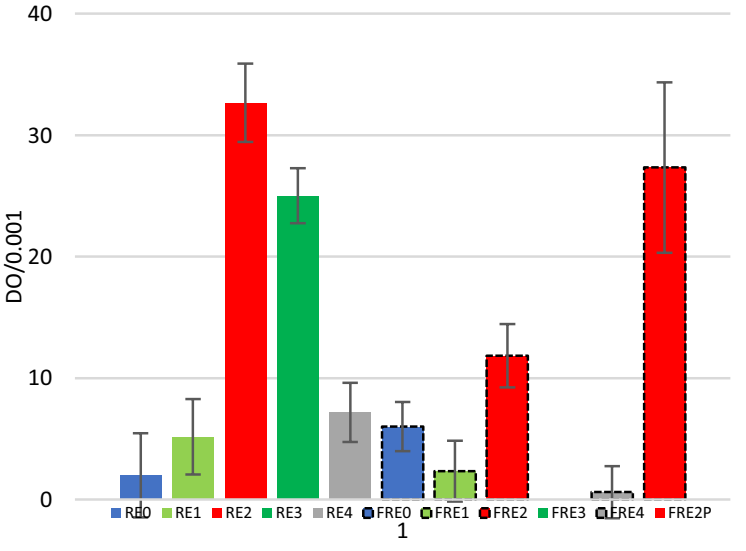

40.

30.

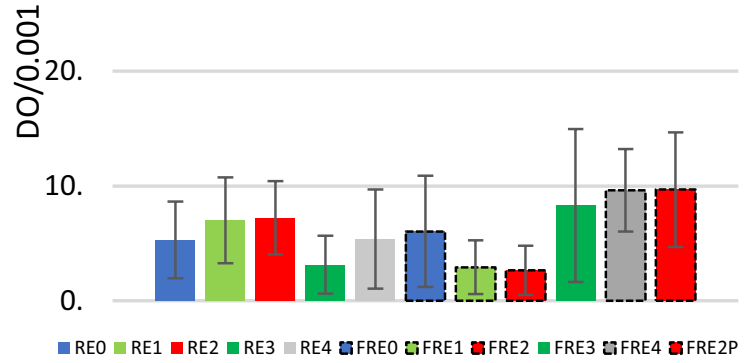

Figure 7. Activités protéases résiduelles des vins rouges (Gamay, 2017) avant (en haut) et après filtration (en bas), après 4 semaines de contact.
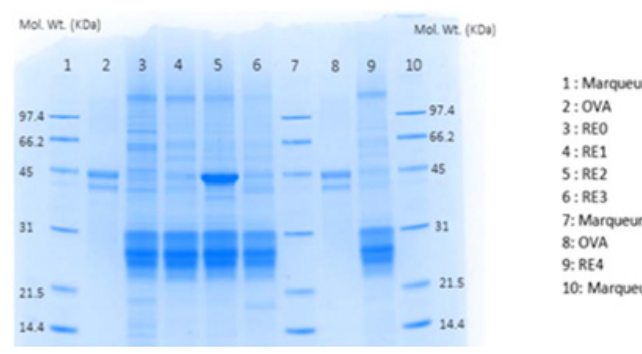

2: OVA

4: REO

4: RE1
S: RE2

6: RE2

7: Marqueur

8: OVA

10: Marqueur

Figure 8. Composition en protéines des vins rouges sans flash pasteurisation (gel SDS-PAGE).

\subsubsection{Electrophorèses (SDS-PAGE)}

Les protéines présente dans les vins des essais ont été analysées par gel d'électrophorèses (SDS-PAGE).

La trop faible concentration en protéines des vins blancs n'a pas permis de mettre en évidence des bandes sur les gels (Résultats non présentés).

Les vins rouges sans flash pasteurisation (Fig. 8) contiennent plus de protéines et laissent apparaitre un nombre important de bandes différentes par rapport aux vins pasteurisés (Fig. 9). Sans flash pasteurisation, il y a assez peu de différences entre les différentes enzymes. A nouveau l'enzyme E2 se distingue par la présence d'une bande intense aux alentours des 45'000 Da devant correspondre à la protéase elle-même.

Les vins rouges flash pasteurisés (Fig. 9) contiennent moins de protéines en quantité et en nombre. La flash pasteurisation contribue aussi à la dénaturation et l'élimination de ces protéines. Encore une fois le vin flash 


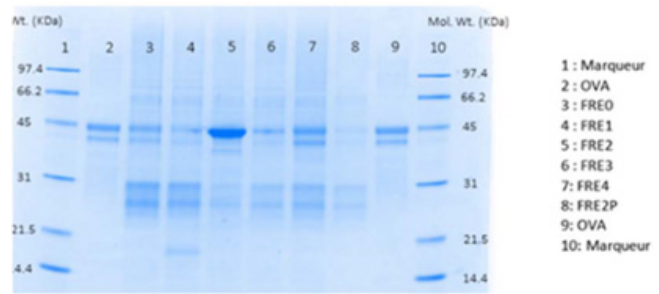

Figure 9. Composition en protéines des vins rouges avec flash pasteurisation (gel SDS-PAGE).

Tableau 2. Caractéristique chromatique des vins.

\begin{tabular}{|l|c|c|c|}
\hline Vin & $\mathbf{L}^{*}(\mathbf{C})$ & $\mathbf{a}^{*}(\mathbf{C})$ & $\mathbf{b}^{*}(\mathbf{C})$ \\
\hline BE0 & $\mathbf{9 9 . 7 4}$ & $-\mathbf{0 . 3 9}$ & $\mathbf{1 . 5 8}$ \\
\hline FBE2 & $\mathbf{9 9 . 8 0}$ & $-\mathbf{0 . 4 3}$ & $\mathbf{1 . 6 7}$ \\
\hline RE0 & $\mathbf{6 6 . 5 6}$ & $\mathbf{3 7 . 5 3}$ & $\mathbf{4 . 3 5}$ \\
\hline FRE2 & $\mathbf{6 8 . 2 9}$ & $\mathbf{3 5 . 2 7}$ & $\mathbf{4 . 2 8}$ \\
\hline
\end{tabular}

Tableau 3. Résultats des tests triangulaires.

\begin{tabular}{|l|c|c|c|}
\hline & $\begin{array}{c}\text { Réponses } \\
\text { retenues }\end{array}$ & $\begin{array}{c}\text { Réponses } \\
\text { exactes }\end{array}$ & Significativité \\
\hline$B E 0 / F B E 0$ & 14 & 4 & 0.7388 \\
\hline$B E 0 / F B E 2$ & 14 & 4 & 0.7388 \\
\hline$B E 0 / B E 2$ & 14 & 5 & 0.5245 \\
\hline REO/FRE2 & 14 & 7 & 0.1495 \\
\hline
\end{tabular}

pasteurisé contenant l'enzyme E2 se distingue. C'est lui qui contient le moins de protéines sauf la bande marquée à 45’000 Da.

\subsubsection{Analyses sensorielles}

Les vins sont dans un premier temps comparés par Napping. Ainsi le dégustateur va regrouper spatialement les modalités où il y a des ressemblances sensorielles.

Les résultats de ce Napping ne nous permettent pas de déterminer des tendances claires. Les vins Flash Pasteurisés se retrouvent dans tous les groupes. Cette technique n'a semble-t-il pas affecté les vins sur le plan sensoriel. Un groupe de vins blancs contient toutes les variantes des enzymes E2 et E3. Ces enzymes étant les plus concentrées en activités protéases, il faut s'y intéresser par la suite afin de confirmer ou infirmer cette observation.

Comme pour le Napping réalisé sur les vins blancs, nous observons des groupes des modalités très différentes. Les résultats du Napping des vins blancs et rouges (Fig. 6) ne sont pas comparables, il n'y pas de tendance particulière qui se forme. Cela amène à penser que les techniques utilisées n'ont pas influencé les vins.

Dans les résultats des deux Napping RE0 et BE0 (témoins sans enzyme) se retrouvent dans des groupes isolés et distincts des autres vins. Afin de savoir si l'enzyme a un impact significatif sensoriellement des tests triangulaires ont été réalisés sur les échantillons qui apparaissent le plus éloignés.

Pour l'ensemble des tests triangulaires (Tableau 3) les échantillons ne sont pas significativement différents même pour des $\alpha=0.10$ (il faudrait 8 valeurs juste pour 14 dégustateurs pour que les différences soient significatives à ce seuil et 10 au seuil de 0.01). En conclusion, ni la flash pasteurisation, ni l'enzymes E2, ni la combinaison des 2 ne semblent affecter significativement les vins d'un point de vue organoleptique.

\section{Conclusion}

Ce travail a permis de mettre en évidence que l'utilisation de protéase peut améliorer la filtrabilité des vins. L'amélioration observée est plus importante avec un test de filtrabilité sur membrane $0.45 \mu \mathrm{m}\left(V_{\max }\right)$ qu'en filtration tangentielle sur membrane céramique $0.2 \mu \mathrm{m}$.

Parmi les quatre préparations enzymatiques commerciales testées seulement deux ont montré une activité protéasique, dans le milieu très inhibant qu'est le vin, et seulement une est capable d'hydrolyser les protéines du vin. Une flash pasteurisation (1 min, $60-70{ }^{\circ} \mathrm{C}$ ) est nécessaire à la dénaturation des protéines $\mathrm{du}$ vin permettant ensuite leur hydrolyse enzymatique. L'addition de protéase E2 avant flash pasteurisation est plus efficace qu'après. La montée en température semble combiner deux actions, la dénaturation des protéines du vin et une meilleure activation des protéases. En même temps, cette flash pasteurisation diminue significativement l'activité protéase résiduelle dans les vins. L'effet activant et dénaturant de la température reste encore à comprendre même s'il est possible qu'à ces températures l'hydrolyse des protéines est plus rapide que la dénaturation des protéases.

La combinaisons flash pasteurisation et filtration tangentielle donne des activités protéasiques résiduelles inferieures au témoin sans enzyme donc pouvant être considérées comme nulles.

Sur les deux vins testés, il n'a pas été possible de mettre en évidence de différences en analyse sensorielle entre les variantes.

Les résultats préliminaires obtenus sont encourageants mais restent à confirmer. Il semble donc important de pouvoir répéter ces expériences sur différents vins avec différentes richesses en protéines. Il reste également en suspens de nombreuses questions notamment quant à l'optimisation du procédé en termes de dose d'enzyme, de temps de contact et de flash pasteurisation ainsi que de niveau de température.

La flash pasteurisation étant déjà utilisé en œnologie et l'échelle choisie dans ces essais (cuves de $250 \mathrm{~L}$ ) sont des éléments rassurants quant à la possibilité de transfert de cette technique en cave.

Ce projet s'est déroulé à Changins haute école de viticulture et onologie (Nyon, Suisse), en partenariat avec Oenoppia regroupement de producteurs de produits œnologiques (Paris, France). Le vin a été gracieusement fourni par la cave Schenk SA (Rolle, Suisse). Le filtre tangentiel a été prêté par l'Institut Universitaire de la Vigne et du Vin de Dijon. Le projet a été sélectionné pour l'OIV Research Grant 2017 et réalisé par Romain Cahuzac dans le cadre de son stage de Master de l'Institut des Sciences de la vigne et du vin de Bordeaux. Nous remercions tous ces partenaires pour leurs contributions.

\section{Références}

[1] J. Ducruet, Revue des Oenologues 89, 25 (1998)

[2] M. Rao, A. Tanksale, M. Ghatge, V. Desphpande, Microbiol. Mol. Biol. Rev. 94, 597 (1998)

[3] L. Theron, B. Divol, Appl. Microbiol. Biotechnol. 98, 8853 (2014) 
[4] I. Benucci, M. Esti, K. Liburdi, Biotechnol. Prog. 31, 48 (2015)

[5] K. Yokotsuka, K. Nozaki, T. Takayanagi, Am. J. Enol. Vitic. 45, 410 (1994)

[6] H. Fontoin, C. Saucier, P.-L. Teissedre, Y. Glories, Food Qual. Preference 19, 286 (2008)

[7] E. Carvalho, N. Mateus, B. Plet, I. Pianet, E. Dufourc, V. De Freitas, J. Agric. Food Chem. 54, 8936 (2006)

[8] L.S. Lagace, L.F. Bisson, Am. J. Enol. Vitic., 147 (1990)

[9] K.F. Pocock, P.B. Hoj, K.S. Adams, M.J. Kwiatkowski, E. Waters, Aust. J. Grape and Wine Res., 56 (2003)

[10] L. Conterno, C. Delfini, J. Wine Res. 5, 113 (1994)
[11] M. Dufrechou, F. Sauvage, B. Bach, A. Vernhet, J. Agric. Food Chem. 58, 10209 (2010)

[12] M. Marangon, S. Van Sluyter, E. Robinson, R. Muhlack, H. Holt, P. Haynes, E. Waters, Food Chem. 135, 1157 (2012)

[13] E. Robinson, N. Scrimgeour, M. Marangon, R. Muhlack, P. Smith, P. Godden, D. Johnson, Wine Vitic J. 27, 24 (2012)

[14] S. Buelga, C.A.-A., Wine Chem. Biochem., 529 (2009)

[15] L. Theron, M. Bely, B. Divol, J. Sci. Food Agric. 97, 3584 (2017)

[16] A.-C. Sivestri, J. Sabatier, J. Ducruet, J. Int. Sci. Vigne Vin. 47, 213 (2013) 